\title{
CDYL and REST — a silent team
}

RE1-silencing transcription factor (REST) is a repressor that, along with a number of co-repressors, can modify histones and shut down transcription. It is also a tumour suppressor gene, but the mechanism of how it prevents tumorigenesis has been unclear. Yang Shi and colleagues

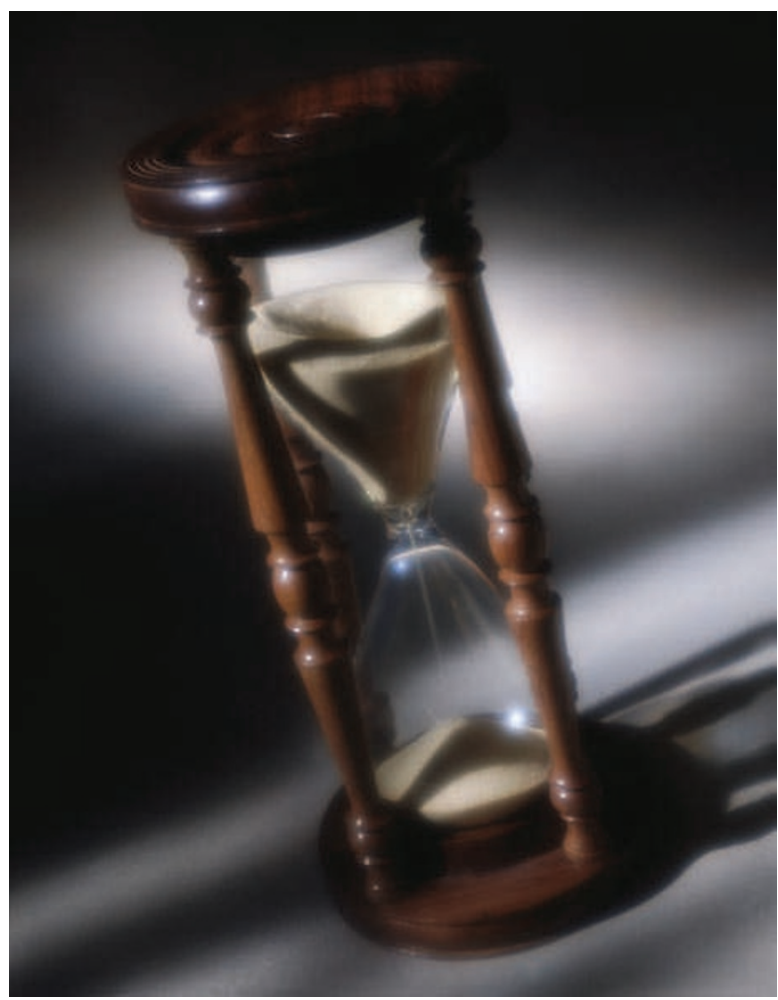

now show that chromodomain Y-like protein (CDYL) is required to mediate REST-induced suppression of the neurotrophic tyrosine kinase receptor type 3 (NTRK3, also known as TRKC).

Yang Shi and colleagues were investigating the function of the enigmatic CDYL. Affinity purification of a Flag-HA-tagged CDYL from HeLa cell nuclear extracts and glycerol gradient sedimentation showed that REST is a component of a complex that includes CDYL, a number of methyltransferases and the histone methylase G9a. REST is known to recruit G9a and further investigations showed that CDYL acts as a bridging protein that enables REST to enlist G9a both in vitro and in vivo. The authors also found that RNA interference knockdown of CDYL in TLM-HMEC cells resulted in the loss of CDYL and G9a from RE1 sites in both TRKC and NPTXR and reduced methylation of histone $\mathrm{H} 3$ lysine 9 (H3K9) at these sites. The retention of REST and histone $\mathrm{H} 3$ at these sites in the absence of CDYL indicates that CDYL is a REST co-repressor that enables recruitment of G9a and subsequent $\mathrm{H} 3 \mathrm{~K} 9$ methylation.

What are the biological consequences of these interactions? RNA interference knockdown of any of CDYL, REST or G9a resulted in transformation of TLM-HMEC cells with the concomitant loss of REST-mediated repression of TRKC. Importantly, knockdown of other REST co-repressors had no effect on TRKC expression, indicating that the CDYL-G9a-REST complex might specifically function to repress TRKC and prevent cellular transformation.

Interestingly, the authors also found that CDYL might restrict the transformation of cervical cells expressing oncoproteins from highrisk strains of human papilloma virus (HPV). The CDYL locus resides at $6 \mathrm{p} 25$, a region associated with loss of heterozygosity and poor prognosis in cervical carcinomas. Two different short hairpin RNAs that targeted CDYL in squamous epithelial cells transfected with HPV16 E6 and E7 increased the efficiency of colony formation compared with cells transfected with E6 and E7 alone.

These findings provide insight into how repressors such as CDYL and REST facilitate tumour suppression through the regulation of specific histone-modifying complexes. Nicola McCarthy

ORIGINAL RESEARCH PAPER Mulligan, P. et al. CDYL bridges REST and histone methyltransferases for gene repression and suppression of cellular transformation. Mol. Cell 32, 718-726 (2008) 